\title{
Prevalence of urinary tract infection, its causative agents and antibiotic sensitivity pattern : A study in Northern International Medical College Hospital, Dhaka
}

${ }^{1}$ Prof. Dr. B H Nazma Yasmeen MBBS, MCPS, MD (Paed) Head, Dept. of Paediatrics Northern International Medical College

${ }^{2}$ Saad Islam

2nd year student

Dept. of Computer Science \& Engineering North South University, Dhaka

${ }^{3}$ Saba Islam

4th year student

Dept. of Microbiology

North South University, Dhaka

${ }^{4}$ Prof. Dr. Md. Moyez Uddin MBBS, D.Bact; M.Phil (Microbiology) WHO trained (UK, Japan, Thailand, India) Head, Dept. of Microbiology Northern International Medical College, Dhaka

${ }^{5}$ Dr. Rowshan Jahan MBBS, DCH, MD (part-III) BICH Medical Officer Dhaka Shishu (Children) Hospital

\section{Correspondence}

Prof. Dr. B H Nazma Yasmeen

Head, Dept. of Paediatrics

Northern International Medical

College, Dhaka

prof.nazma.yasmeen@gmail.com

\section{B H N Yasmeen ${ }^{1}$, S Islam², S Islam ${ }^{3}$, M M Uddin ${ }^{4}$, R Jahan ${ }^{5}$}

Abstract

Background : Urinary tract infection (UTI) is one of the most important causes of morbidity and mortality in the developing countries like Bangladesh. Antimicrobial agents are the frequently used drug for its treatment. Periodic evaluation of antimicrobial activity of different antibiotics is essential as the pattern of antibiotic sensitivity may vary over periods. Increasing antibiotic resistance among urinary pathogens to commonly prescribed drugs has become a global reality today.

Objective : To determine the prevalence and to find out the causative agents of UTI and their antibiotic sensitivity pattern among suspected UTI patients attending Northern International Medical College Hospital (NIMCH), Dhaka.

Methods : A retrospective study was conducted at Northern International Medical College Hospital, Dhaka, Bangladesh by analyzing the records of urine samples collected for culture and sensitivity tests over a period of six months (January 2014 to June 2014). Of the total 878 clean catch mid-stream urine samples collected from suspected cases of UTI patients of all ages and both sexes. Urine specimens were cultured for isolation of microbial agents of urinary tract infection. The isolated bacteria were identified using biochemical test. The diffusion susceptibility test was used to determine susceptibility of bacterial agents to antibiotics. Computerized data was collected from Microbiology department of NIMCH and analyzed by Microsoft Excel Version 2013.

Results : In this study, $182(20.73 \%)$ out of 878 urine sample were positive for pathogenic organisms. Of the various pathogenic organisms isolated, Escherichia coli constituted for $85.16 \%$ followed by Pseudomonas sp, Acinatobactersp, Group D Streptococcus, Staphylococcus aureus, Klebsiellasp, Enterobactersp and others. E.coli was found to be most sensitive to Imipenem, Amikacin and Meropenem and resistant to most commonly used oral drugs like Azithromycin, Cefexime, cotrimoxazole and Ciprofloxacin and Levofloxacin.

Conclusion : Mainly Gram negative bacilli is responsible for UTI and most frequent isolated bacteria was E-coli. The most effective antibiotics were Imipenem, Amikacin, Meropenem,all of them are parentral. Majority E-coli were resistant to commonly used oral drugs like Azithromycin, Cefexime, cotrimoxazole and Ciprofloxacin. Therefore the choice of antibiotic therapy in UTI should be depends on the local sensitivity pattern of the infecting organisms.

Key words : Urinary Tract Infection, Isolated Bacteria, Antibiotic sensitivity

\section{Introduction}

UTI is the most common bacterial infection accounting for $25 \%$ of all infections. It is one of the most important causes of morbidity and also the second most common cause of hospital visit. $^{1}$

It occurs in all populations and ages from the neonate to the geriatric age group. ${ }^{2}$ However, infection is most common in women, especially sexually active women. ${ }^{3}$ Women are more susceptible than men, due to several clinical factors including anatomic difference, hormonal effects and behavioral pattern. ${ }^{4}$

In Bangladesh urinary tract infection (UTI) is also one of the most important causes of morbidity and mortality. This may be attributed to lack of proper research, faulty diagnostic procedures, abuse of chemotherapeutic agents of the people and little or no preventive measures. ${ }^{5}$ The major pathogens causing UTI are E. coli and Pseudomonas spp, Proteus spp, klebsiella spp etc. ${ }^{6}$ Many investigators in Bangladesh also showed that women were the usual victims of the urinary pathogens. Haqueet al. reported $78.8 \%$ of the $E$. coli infection in women. ${ }^{7}$ Nahar and Selim worked on adult women and reported that $70.9 \%$ organisms were found to be $E$. coli. ${ }^{8}$

The alarming phenomenon is that UTI does not restrict itself to the urinary tract only rather it can spread. UTI infections usually cause inflammation of the affected tissues of the urethra (urethritis) and urinary bladder. The most significant danger from lower urinary tract infections it can affect the kidney (causing 
pyelonephritis) and develop bladder infections subsequently. 9 Bacteria carried by blood stream can also infect the kidney and the infections can be very difficult to eradicate, are often chronic, and lead to marked damage of the kidney.

In case of children UTI may lead to life threatening complications like sepsis and renal scaring. Renal scaring is the most common cause of hypertension in later childhood and renal failure in adulthood. ${ }^{11}$ Recognition of UTI in children should be made as early as possible to prevent these complications. ${ }^{11}$ Therefore, investigations for early diagnosis of UTI are of outmost importance. ${ }^{12}$

The isolation of bacteria in urine in a significant quantities consistent with infection. Therefore isolation of organisms causing UTI and their antibiotic susceptibility is very essential for their appropriate management. As Because Antibiotics are the specific treatment for a urinary tract infection though resistance to antibiotics is highly prevalent in bacterial isolates all over the world, particularly in developing countries. ${ }^{13}$

In Bangladesh, use of antibiotics by medical practitioners is rampant resulting in increase in resistance to available antibiotics. Random and extensive use of broad spectrum of antibiotics contributed to changes in the microbiological and antibiotic susceptibility patterns of pathogens isolated from UTI. Therefore for effective management of these infections, selection of antibiotics should be based on antibiotic susceptibility pattern. But it is often hampered by the lack of adequate facilities for proper microbial isolation as well as for their antimicrobial susceptibility testing. ${ }^{14}$

Besides this, periodic evaluation of antimicrobial activity of different antibiotics is essential as the pattern of antibiotic sensitivity may vary over periods. ${ }^{15}$ Increasing antibiotic resistance among urinary pathogens, especially $E$. coli, to commonly prescribed drugs like cotrimoxazole has become a global reality. ${ }^{16}$

To provide appropriate antibiotic treatment, physicians need to know local patterns of microbial susceptibility and cost effectiveness for proper drug selection. ${ }^{17}$ Therefore, the purpose of this study was to determine the prevalence of UTI and to identify the prevalent microorganisms and the recent trend of their antimicrobial susceptibility patterns of suspected UTI patients attending at Northern International Medical College Hospital.

\section{Materials and methods}

A retrospective study was conducted to determine the prevalence and to find out the causative agents of UTI and their antibiotic sensitivity pattern among suspected UTI patients attending Northern International Medical College Hospital (NIMCH). This study was done at the Paediatrics Department of NIMCH Dhaka, Bangladesh by analyzing the Computer database records (Kept in the Microbiology Department of $\mathrm{NIMCH}$ ) of urine samples collected for culture and sensitivity tests for a period of six months (January to June 2014)

Total 878 suspected UTI patients from birth to more than 70 years of age and both sexes were included in this study. Clean catch midstream urine samples were collected into a wide mouthed sterile container. Then urine inoculated on MacConkey's and Blood Agar media using calibrated platinum loop following standard bacteriological technique and incubated at $37^{\circ} \mathrm{C}$ for $24-48$ hours.After 24 to 48 hours the plate were examined for bacterial pathogen. Pure bacterial colony counting 100,000 or more was considered as significant and was subjected to identification based on colony characters and biochemical tests.

In this study culture was considered Positive when the culture of a single microorganism found at a concentration of $>10^{5}$ colonyforming units (CFU)/ml. Commercially available discs (supplied by HiMedia, India) were used to find the sensitivity of the organism on Mueller-Hinton Agar media after overnight incubation at $37^{\circ} \mathrm{C}$. Test disks used included nitrofurantoin $(300 \mathrm{mcg})$ Amikacin, $(30 \mathrm{mcg})$, levofloxacin $(5 \mathrm{mcg})$, ciprofloxacin (5mcg), cotrimoxazole $(1.25 / 23.75 \mathrm{mcg})$, ceftriaxone $(30 \mathrm{mcg})$, cefotaxime $(30 \mathrm{mcg})$, Imipenem $(10 \mathrm{mcg})$, Meropenem $(10 \mathrm{mcg})$, Azithromycin, (15mcg), Cefexime (15mcg) and Bacitricin $(10 \mathrm{mcg})$, Ceftazidime (30 mcg).

Finally all the data were analyzed with Microsoft Excel version 2013.

\section{Results}

A total of 878 urine samples were collected from suspected UTI patients. The age and sex distribution of patients from whom the urine samples were collected is shown in Figure 1.

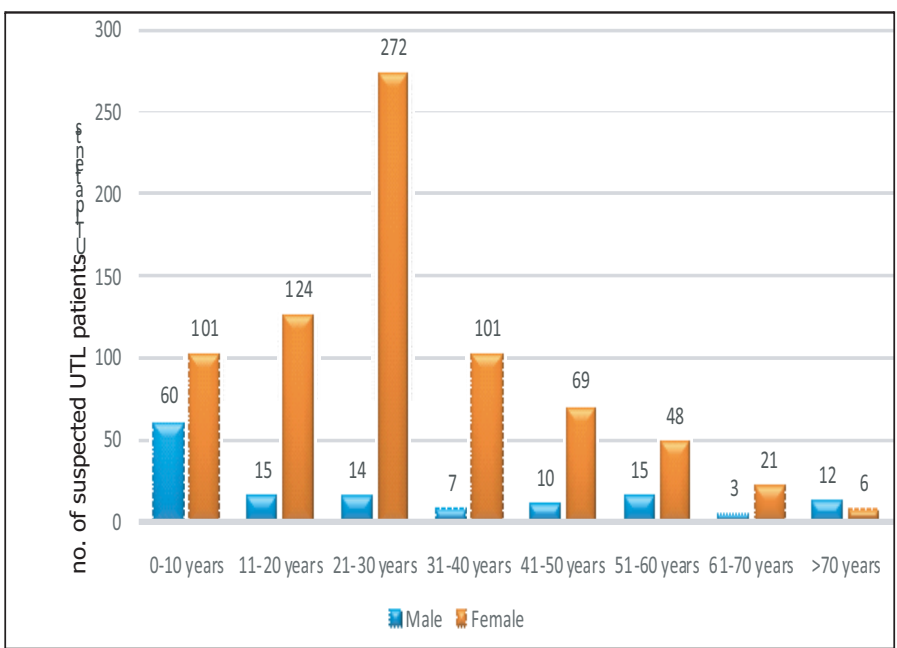

Figure 1 : Age and sex distribution of suspected UTI patients (Total no 878)

Majority of the cases were in the age group of 21 to 30 years and least patients were in more than 70 years of age group. Relatively more number of urine samples were collected from female patient compared with male. 
Of the total 878 urine samples $182(20.73 \%)$ were positive for pathogenic organisms (Figure 2). The types of organisms isolated are shown in Figure 3. Escherichia coli was isolated in $155(85.16 \%)$ of the positive samples. This was followed by Pseudomonas sp 8(4.39\%) and Acinetobactor sp and Group D Streptococcus (2.20\%), Staph. Aureus, Klebsiella sp, Enterobacter $s p$, Salmonella typhi all of these organism were less than $2 \%$. (Figure 3 ).

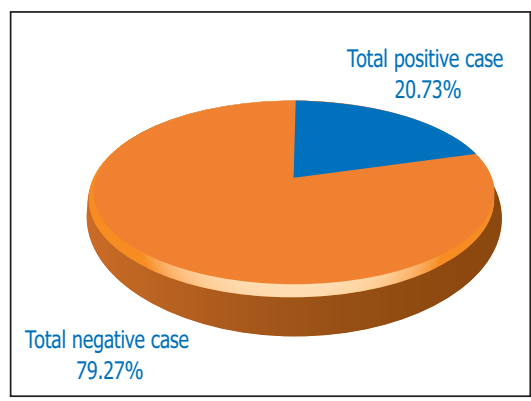

Figure 2: Bacterial growth positive rate in urine samples collected.

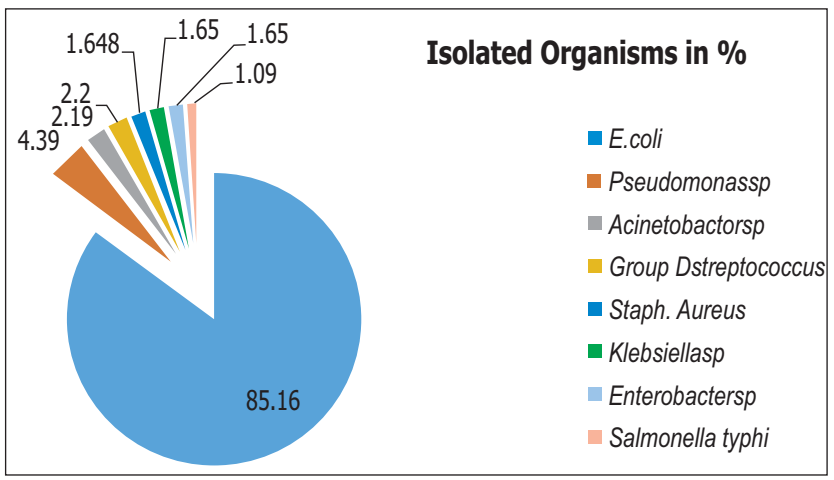

Figure 3 : Age and sex distribution of culture positive UTI patients (Total no182)

In our study majority of growth positive cases were in the age group of 21-30 years, followed by children up to 10 years (Figure 4) The prevalence of UTI (growth positive cases) was recorded higher in females than in males. Females were predominant with UTI showing $164(90.10 \%)$ of urine culture positivity whereas the male subjects showed only $18(9.90 \%)$ of culture positivity.

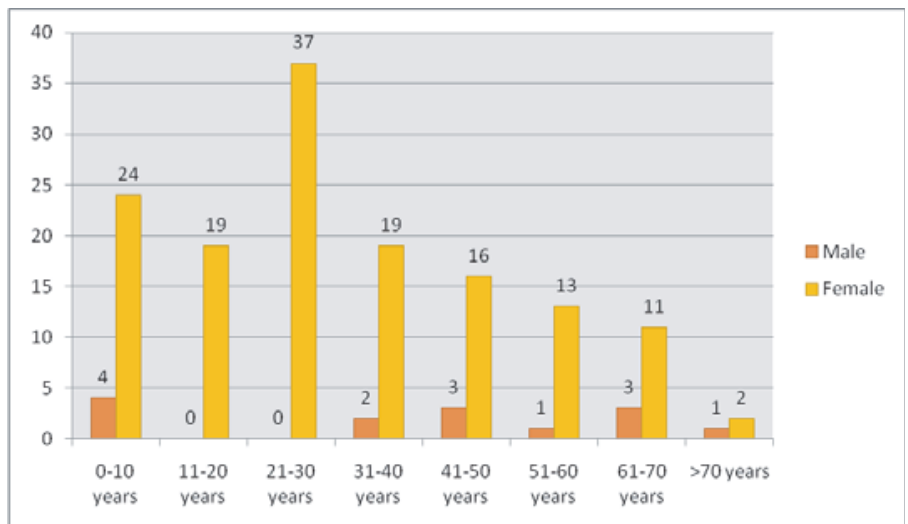

Figure 4 : Pattern of organism isolated from urine culture
E. coli was found to be most sensitive to Imipenem, Amikacin, Meropenem and resistant to most commonly used drugs like Azithromycin Cefexime, cotrimoxazole and Ciprofloxacin and Levofloxacin. Pseudomonas sp was found to be most sensitive to ceftriaxone, cefotaxime, ciprofloxacin and ofloxacin and least sensitive to cephalexin. Klebsiella $s p$ was most sensitive to ofloxacin followed by ceftriaxone and least sensitive to amikacin and nitrofurantoin (Table-1).

Table-1: Antibiotic sensitivity pattern of E. coli

\begin{tabular}{lccccccc} 
Antibiotics & $\begin{array}{c}\text { Isolates } \\
\text { tested }\end{array}$ & \multicolumn{2}{c}{ Sensitive } & Resistance & \multicolumn{2}{c}{ Intermidiate } \\
& & no & $\%$ & no & $\%$ & no & $\%$ \\
Imipenem & 140 & 123 & 87.86 & 10 & 7.14 & 7 & 5.0 \\
Amikacin & 146 & 123 & 84.25 & 8 & 5.48 & 15 & 10.27 \\
Meropenem & 119 & 92 & 77.31 & 27 & 22.69 & 0 & 0 \\
Nitrofurantoin & 147 & 94 & 63.96 & 27 & 18.37 & 26 & 17.67 \\
Ceftazidime & 67 & 39 & 58.21 & 5 & 7.4 & 23 & 34.33 \\
Ceftriaxone & 144 & 84 & 55.33 & 42 & 29.17 & 18 & 12.50 \\
Levofloxacin & 144 & 69 & 47.92 & 59 & 40.97 & 16 & 11.11 \\
Ciprofloxacin & 129 & 60 & 46.51 & 52 & 40.31 & 17 & 13.18 \\
Cotrimoxazole & 143 & 64 & 44.76 & 65 & 45.45 & 14 & 9.79 \\
Cefexime & 125 & 24 & 19.20 & 85 & 68.0 & 16 & 12.8 \\
Azithromycin & 115 & 17 & 14.78 & 76 & 66.08 & 22 & 19.30
\end{tabular}

Pseudomonas $s p$ was found to be most sensitive to all drugs tested i,e 100.0\% sensitive to Nitrofurantoin, Amoxicillin, Amoxycillin Clavulanic Acid, Azithromycin, amikacin, ceftriaxone, levofloxacin cotrimoxazole, Imipenem, Cefexime, Meropenem Bacitricin and 50\% resistant only to ciprofloxacin (Figure 5).

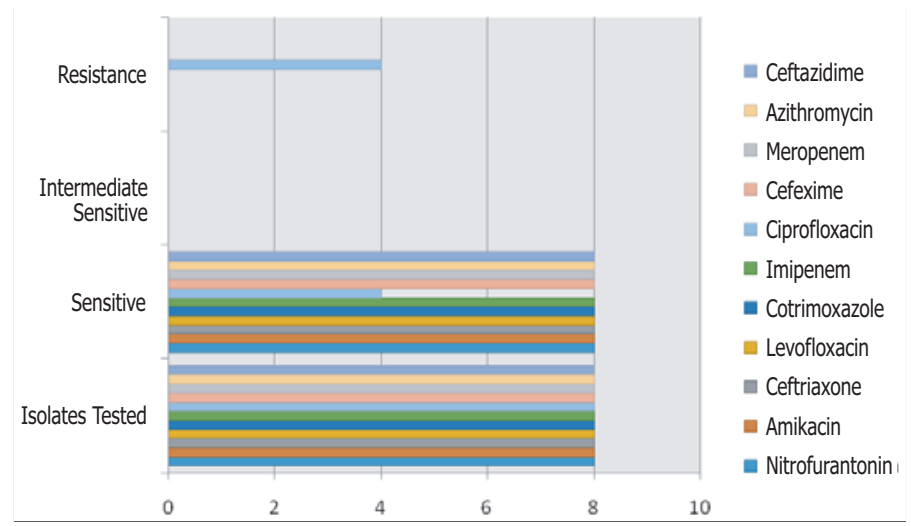

Figure 5: Antibiotic sensitivity pattern of Pseudomonas sp

\section{Discussion}

UTI is a common clinical problem in both the community and health care associated settings. Epidemiologically urinary tract infections account for seven million office visits and one million emergency department visits, resulting in 100,000 hospitalizations yearly, making them the most common bacterial 
infections in outpatient and emergency department setting. Financially, the estimated annual cost of UTI is significant, at approximately $\$ 1.6$ billion. ${ }^{18}$

The prevalence of UTI varies according to sex and age. ${ }^{19}$ It has been usually observed that UTI most commonly occurs in females and up to one-third of all women experience a UTI at some point during their lifetimes. ${ }^{20}$

In this study age and sex distribution of majority of the cases from whom the urine samples collected were in the age group of 21 to 30 years. Every month relatively more number of samples were obtained from female patients compared with males except patients more than 70 years of age. A total of 878 urine specimens were collected from patients suspected of having UTI, out of which $182(20.73 \%)$ showed significant bacterial growth and were included in this study. The frequency is close to the incidence reported by Ahmed and Avasarala was $12.7 \%,{ }^{21}$ but is higher than the study of Singh MM et al. who reported $4.2 \%$ UTI in a community based study. ${ }^{22}$ Patients in Bangladesh usually see a doctor after experiencing severe health complications for a particular disease condition. Therefore, Bashar et al. and Rahman et al. reported higher frequency of UTI i.e., $27 \%$ and $24.14 \%$ respectively in hospital or clinic based study. 23,24

In our study majority of growth positive cases were in the age group of 21-30 years, followed by children up to 10 years. This results are more or less consistent with other studies. ${ }^{5,25}$ The prevalence of UTI (growth positive cases) was recorded higher in females than in males. Females were predominant with UTI showing $90.10 \%$ of urine culture positivity whereas the male subjects showed only $9.90 \%$ of culture positivity. Among the 182 isolates obtained, 164 were from females while only 18 were from males. Similar observations were also recorded by Astal et al. ${ }^{26}$ and Khalifa et al. ${ }^{27}$ In Bangladesh, Begum et al. reported $16.4 \%$ UTI in the female garments workers of Dhaka City. ${ }^{28}$

Bacteriological studies usually reveal the involvement of gram negative enteric organisms that commonly cause urinary tract infections, such as E. coli, the Klebsiella species, and the Proteus species. ${ }^{29}$ The most common organism implicated in UTIs $(80-85 \%)$ is E. coli. ${ }^{30}$

Similarly, the predominant number of pathogens isolated in our study were gram negative bacilli rather than gram positive pathogens. In this study Escherichia coli constituted the largest group with a prevalence of $85.16 \%$, then Pseudomonas sp only 4.40\% Acinetobactor sp and Group D Streptococcus (2.20\%), Staph. Aureus, Klebsiella sp, Enterobacter sp, Salmonella typhi all of these organism were less than $2 \%$.

Other investigators (Basar et al. and Saber et al. also reported higher association of E. coli $(66.67 \%$ and $77.8 \%$ cases respectively) in UTI patients. ${ }^{23,31}$ The study conducted in 2014 in Lahore; Pakistan shows the prevalence of UTI with the highest prevalence of E-coli ( $80 \%)$ followed by Staphylococcus aureus $(9.4 \%)$, Proteus species (5.4\%) and Pseudomonas species (5.2\%). ${ }^{14}$ In most of the studies Escherichia coli was the prevalent organism in UTI. ${ }^{25,30}$

The pattern of antimicrobial resistance of the micro-organisms causing UTI infections vary in their susceptibility to antimicrobials from place to place and from time to time. ${ }^{33}$ In this study E. coli was observed to be sensitive to almost all the antibiotics tested, though to varying degrees. E. coli was found to be most sensitive to parentral Imipenem (87.86\%), Amikacin $(84.25 \%)$, Meropenem $(77.31 \%)$ and resistant to most commonly used oral drugs like Cefexime (68\%), Azithromycin (66.08\%) cotrimoxazole (45.45\%) and Ciprofloxacin (40.31\%) and Levofloxacin (41\%).

Antibiotic abuse and practicing incomplete antibiotic regimen has considerably promoted the dissemination of multidrug resistant bacteria. ${ }^{34}$ Our study showed that E. coli had lower resistance for less commonly used drugs like imipenem, meropenem, amikacin and nitrofurantoin. This finding is supported by the study of Sharmin et al. which reported a good sensitivity for imipenem, ceftazidime and amikacin against UTI-isolates of E. coli in Bangladesh. ${ }^{35}$ Encarnacion A R also reported sensitivity of the UTI-isolates of E. coli for amikacin in Philippines. ${ }^{36}$ This finding suggests the use of drugs that are less commonly prescribed by practitioners for arresting the pathogens in UTI patients may be beneficial.

The antibiotic susceptibility patterns of Pseudomonas $s p$ were also variable. In this study Pseudomonas $s p$ less prevalent bacteria with a susceptibility of $100 \%$ sensitive to almost all the antibiotics tested, both oral and parentral. Even though Pseudomonas sp were the predominant organisms in nosocomial UTI ${ }^{17}$ Gram negative bacilli were found to be highly sensitive to ciprofloxacin (62\%) than to ofloxacin (23\%). Ciprofloxacin is thus clearly useful against polyresistant species such as Pseudomonas aeroginosa. ${ }^{37}$ But this finding is not consistent with our study. In our study Pseudomonas sp 50\% resistant to only one drug i,e Ciprofloxacin.

This study also showed that E. coli and Pseudomonas sp both of them are nearly $50 \%$ resistant to Ciprofloxacin. Therefore, Healthcare practitioners must think critically before prescribing the commonly used drugs Ciprofloxacin to a UTI patient.

\section{Conclusion}

UTI among female is more prevalent and the most predominant uropathogen was $E$ coli. Most effective antimicrobial agents for $E$ coli are parentral Imipenem, Amikacin and Meropenem. It is resistant to most commonly used oral drugs like Cefexime, Azithromycin, cotrimoxazole and Ciprofloxacin and Levofloxacin. 
Therefore the choice of antibiotic therapy should be integrate the local sensitivity pattern of the infecting organisms.

The periodic evaluations of predominant organisms and their antibiotic susceptibility pattern are necessary as it is changing with time. The resistant pattern to antibiotics is very important to help the clinician to choose right empirical treatment before the final bacterial result of urine culture of a patient is available or where the lab facility is not available.

\section{References}

1. Ronald AR. The natural history of urinary infection in adults. Med Clin North Am. 1991; 75:299-312p.

2. Kunin CM. Urinary tract infections in females. Clin Infect Dis 1994;18:1-12

3. Karki A, Tiwari BR, Pradhan SB. Study of bacteria Isolated from Urinary Tract Infections and Their Sensitivity Pattern. Journal of Nepal Medical Association 2004; 43:200-203.

4. Hooton TM. Pathogenesis of urinary tract infections: an update. J Antimicrob Chemother. 2000; 46 Suppl 1: 1-7.

5. Hamida K, Fahmida M, Shafiullah A. Z. M., Farhana M. Prevalence and comparative likelihood of urinary tract infection among female out patients in BSMMU. Bangladesh J. Zool. 2012 40(2): 231-239

6. Basnet BB, Acharya K, Karmacharya N, et al. Journal of Nepal Association for Medical Laboratory Sciences 2009; 10:47-52p.

7. HAQUE, N., KIBRIA, G. and BEGUM, S.N. Prevalence of urinary tract infection in adult women. B.M.R.C. 1976. Bull.2:75-85

8. NAHAR, N., HUQ, F. and SELIM, K. Prevalence of bacteriuria in adult Bangladeshi Females 1989 Bang. J. Microbiol. 6(2): 37-42.

9. Nahar S.I, khanum $\mathrm{H}$, Shimusaki K.. Occurrence of Escherichia coli among the women of Dhaka city. ARPN J. Agri. And Bio. Sci2010. 5(6): 68-73.

10. Warren J, Mc Isaac, Rahim Moineddin, et al. Uropathogen antibiotic resistance in adult women presenting to family physicians with acute uncomplicated cystitis. Can J Infect Dis Med Microbiol. September/October 2004; 15(5).

11. Chon $\mathrm{CH}$, Lai FC, Shorthffe LM. Pediatric urinary tractinfections. PediatrClin North Amer 2001; 48: 1447-59.

12. Watson AR. Disorders of the urinary syastem. In: Campbell AG, McIntosh N, editors. Forfar and Arneil's Textbook of Pediatrics. Churchill Livinstone 1998: 949-56.

13. Lamikanra A, Okeke IN. A study of the effect of the urban/rural divide on the incidence of antibiotic resistance in Escherichia coli. Biomed Lett 1997;55:91-7.

14. Ikeh KI. Methicillin-resistant Staphylococcus aureus (MRSA) at Jos University Teaching Hospital. Afr J ClinExpMicrobiol 2003;4:52-5.

15. Jones RN, Thornsberry C. Cefotaxime: a review of in vitroantimicrobial properties and spectrum of activity. Rev Infect Dis 1982; 4: 5300-15.

16. Manges AR, Johnson JR, Foxman B, O'Bryan TT, FullertonKE, Riley LW. Widespread distribution of urinary tract infections caused by a multidrugresistant Escherichia coli clonal group. New Engl J Med 2001; 345: 1007-13

17. Kurin,CM Detection prevention management of urinary tract infections 1997. $5^{\text {th }}$ Edn. (Baltimore Williams and Wilkins) $128-64$

18. Joseph TD. Urinary Tract Infections and Prostatitis. In Wells BG, Schwinharmmer TL, Hamilton CW, editors. Pharmacotherapy Handbook. 7th ed. New York: McGraw-Hill; 2008; 493-503.
19. Kosokai N Y, Kumaoto T, Hirose N, TankaY, Ltikichi $S$, Sigeta $Y$ et al.Comparative studies on activities of antimicrobial gaunt against causative organisms isolated from urinary tract infection of 1987.11 Background of patients. Japan J.Antiriot,1990.43:954-67.

20. Palac DM. Urinary tract infection in women. A physician's perspective, 1986. p. $17-25$.

21. Ahmed SM, Avasarala AK. Urinary tract infections (UTI) among adolescent girls in rural Karimnagar district, AP - K.A.P. STUDY. Indian J PrevSoc Med. 2008;39(1 2):67-70.

22. Singh MM, Devi R, Garg S, Mehra M. Effectiveness of syndromic approach in management of reproductive tract infections in women. Indian J Med Sci. 2001;55(4):209-214. [PubMed]

23. Bashar MA, Ahmed MF, Rahman SR, Gomes DJ. Distribution and Resistance Trends of Escherichia coli from Urinary Tract Infections Isolated in Dhaka City. Ban J Med Sci. 2009;15(2):93-98.

24. Rahman F, Chowdhury S, Rahman MM, Ahmed D, Hossain A. Antimicrobial Resistance Pattern of Gram-negative Bacteria Causing Urinary Tract Infection. S J Pharm Sci. 2009;2(1):44-50.

25. Bhowmick B K, Rashid $H$. Prevalence and antibiotic susceptibility of E coli Isolated fromUrinary tract infections (UTI) in Bangladesh. Pak.J.Biol.Sci. 2004,7 (5):717-720

26. Astal Z, Sharif F, Manama A. Antibiotic resistance of bacteria associated with community-acquired urinary tract infections in the southern area of the Gaza Strip. J Chemother 2002;14:259-64

27. Khalifa BHA, Khedher M. Epidemiological study of Klebsiella spp. uropathogenic strains producing extended-spectrum betalactamase in a Tunisian University Hospital, 2009. PatholBiol (Paris) 2012;60:e1-5

28. Begum N, Mamoon ABA, Hossain M, Begum N, Chowdhury SA, Rahman MF. UTI among female workers in a selected garment industry of Dhaka city: A cross sectional study. The ORION Medical Journal. 2006;23:325-327

29. Bova JG, Potter JL, Arevalos E, Hopens T, Goldstein HM, Radwin HM. Renal and perirenal infection: to he role of computerized tomography. J Urol. 1985;133:375-8. [PubMed]

30. Nicolle LE. Resistant pathogens in urinary tract infections. J Am GeriatrSoc 2002; $50:$ S230-5

31. Saber MH, Barai L, J Haq A, Jilani MSA, Begum MJ. The Pattern of Organism Causing Urinary Tract Infection in Diabetic and Non Diabetic Patients in Bangladesh. Bangladesh J Med Microbiol. 2010;04(01):6-8.

32. Sabir $S$, Anjum AA, Ijaz $T$, Ali MA, Khan MR, Nawaz M. Isolation and antibiotic susceptibility of $E$. coli from urinary tract infections in a tertiary care hospital. Pak J Med Sci 2014; 30(2):389-392.

33. Banerjee S, Padmashri VP. The Study of Urinary Tract Infections and Antibiogram of Uropathogens in and around Ahmadnagar, Maharashtra. Int J Infect Dis ISSN: 1528-8366; 2011, Vol. 9 No. 1

34. Lee MG, Henry GL. Drug availability in Jamaica. West Indian Med J. 1989;38(2):105-109. [PubMed]

35. Sharmin S, Alamgir F, Fahmida, Saleh AA. Antimicrobial sensitivity pattern of uropathogens in children. Bangladesh J Med Microbiol. 2009;03(01):18-22. 2009.

36. Encarnacion AR. Pathogens Causing Urinary Tract Infection and Their Resistance Patterns among Pediatric Patients in Chong Hua Hospital (January 2003 to June 2005) PIDSP Journal. 2012;13(1):37-43.

37. Notowicz A, Stolz E, van Klingeren B. A double blind study comparing two dosages of enoxacin for the treatment of uncomplicated urogenital gonorrhoea. J AntimicrobChemother. 1984;14:91-4. [PubMed] 